\title{
SOME TRACE INEQUALITIES FOR OPERATORS
}

\author{
XINMIN YANG
}

(Received 23 September 1992)

Communicated by B. Mond

\begin{abstract}
In this paper, we obtain some trace inequalities for arbitrary finite positive definite operators. Finally an open question is presented.

1991 Mathematics subject classification (Amer. Math. Soc.): 47A63.

Keywords and phrases: trace inequality, positive definite operators.
\end{abstract}

In 1978, after giving some trace inequalities for positive definite matrices, R. Bellman brought attention to two open questions [1]. One of the questions asks: Is there a matrix analogy of the arithmetic mean - geometric mean inequality (for positive definite matrices)? Y. Yang [4] proved that the answer to the above question is affirmative for two positive definite matrices. Recently Dinesh Singh [3] generalized the result of Yang to infinite-dimensional spaces.

In this paper, we generalize the trace inequality in [3] from two positive definite operators to an arbitrary finite number of positive definite operators.

Throughout, $C_{p}(1 \leq p<\infty)$ stands for the class of all bounded operators $A$ on an infinite-dimensional separable Hilbert space $H$, such that $\sum_{n=1}^{\infty}\left|\left\langle A e_{n}, e_{n}\right\rangle\right|^{p}<\infty$ for each orthonormal basis $\left\{e_{n}\right\}_{1}^{\infty}$ in $H$. Let $\left\{e_{n}\right\}$ be any orthonormal basis in $H$. Let $\operatorname{Tr}: C_{1} \rightarrow \mathbb{C}$ (complex numbers) be defined by

$$
\operatorname{Tr}(A)=\sum_{n=1}^{\infty}\left\langle A e_{n}, e_{n}\right\rangle
$$

It is easy to see that $\operatorname{Tr}$ is independent of $\left\{e_{n}\right\}$ [2, Lemma 2.2.1], and since $C_{1}$ consists of compact operators [2, Theorem 2.1.6], $\operatorname{Tr}(A)$ is the sum of the eigenvalues of $A$.

(C) 1995 Australian Mathematical Society $0263-6115 / 95 \$ A 2.00+0.00$ 
Furthermore, $\operatorname{Tr}$ defines an inner product on $C_{2}$ given by

$$
\langle A, B\rangle=\operatorname{Tr}\left(B^{*} A\right)
$$

where $B$ is the adjoint of $B$. This inner product makes $C_{2}$ into a Hilbert space [2, Theorem 2.4.2]. Clearly $C_{1}$ is contained in $C_{2}$.

We now state and prove our results as following.

LEMMA 1. Let $A$ be a positive definite operator in $C_{1}$ and $B$ be a operator in $C_{1}$. Then

$$
\operatorname{Tr}(A B)=\operatorname{Tr}(B A)
$$

PROOF. Choose an orthonormal basis $\left\{e_{n}\right\}_{1}^{\infty}$ of $H$ such that each $e_{n}$ is an eigenvector for $A$ with corresponding eigenvalue $\alpha_{n}$. Since $A>0$, each $\alpha_{n}>0$. Let $\beta_{n}=$ $\left\langle B e_{n}, e_{n}\right\rangle$. Then

$$
\begin{aligned}
\operatorname{Tr}(A B) & =\sum_{n=1}^{\infty}\left\langle A B e_{n}, e_{n}\right\rangle=\sum_{n=1}^{\infty}\left\langle B e_{n}, A e_{n}\right\rangle \\
& =\sum_{n=1}^{\infty} \alpha_{n}\left\langle B e_{n}, e_{n}\right\rangle=\sum_{n=1}^{\infty} \alpha_{n} \beta_{n}, \\
\operatorname{Tr}(B A) & =\sum_{n=1}^{\infty}\left\langle B A e_{n}, e_{n}\right\rangle=\sum_{n=1}^{\infty}\left\langle A e_{n}, B^{*} e_{n}\right\rangle \\
& =\sum_{n=1}^{\infty} \alpha_{n}\left\langle e_{n}, B^{*} e_{n}\right\rangle=\sum_{n=1}^{\infty} \alpha_{n}\left\langle B e_{n}, e_{n}\right\rangle \\
& =\sum_{n=1}^{\infty} \alpha_{n} \beta_{n} .
\end{aligned}
$$

Hence (1) is proved.

By the Cauchy-Schwartz inequality, we have

LEMMA 2. Let $A, B$ be two operators in $C_{1}$; then

$$
\operatorname{Tr}(A B) \leq|\operatorname{Tr}(A B)| \leq \sqrt{\operatorname{Tr}\left(A A^{*}\right)} \cdot \sqrt{\operatorname{Tr}\left(B B^{*}\right)} .
$$

LEMMA 3. ([3]). Let $A, B$ be two positive definite operators in $C_{1}$; then

$$
\operatorname{Tr}(A B)<\operatorname{Tr}(A) \cdot \operatorname{Tr}(B) .
$$

LEMMA 4. Let $A_{i}(1 \leq i \leq m)$ be positive definite operators in $C_{1}$; then

$$
\operatorname{Tr}\left\{\left(A_{1} A_{2} \cdots A_{m}\right)\left(A_{1} A_{2} \cdots A_{m}\right)^{*}\right\}<\prod_{i=1}^{m} \operatorname{Tr}\left(A_{i}^{2}\right)<\prod_{i=1}^{m}\left(\operatorname{Tr}\left(A_{i}\right)\right)^{2} .
$$


PROOF.

$$
\begin{aligned}
& \operatorname{Tr}\left\{\left(A_{1} A_{2} \cdots A_{m}\right)\left(A_{1} A_{2} \cdots A_{m}\right)^{*}\right\}=\operatorname{Tr}\left\{A_{1}\left(A_{2} \cdots A_{m}\right)\left(A_{2} \cdots A_{m}\right)^{*} A_{1}^{*}\right\} \\
& =\operatorname{Tr}\left\{\left(A_{1}^{*} A_{1}\right)\left(A_{2} \cdots A_{m}\right)\left(A_{2} \cdots A_{m}\right)^{*}\right\} \\
& <\operatorname{Tr}\left(A_{1}^{*} A_{1}\right) \operatorname{Tr}\left\{\left(A_{2} \cdots A_{m}\right)\left(A_{2} \cdots A_{m}\right)^{*}\right\} \\
& <\operatorname{Tr}\left(A_{1}^{2}\right) \operatorname{Tr}\left(A_{2}^{2}\right) \cdots \operatorname{Tr}\left(A_{m}^{2}\right)<\prod_{i=1}^{m}\left[\operatorname{Tr}\left(A_{i}\right)\right]^{2} .
\end{aligned}
$$

The proof is complete.

THEOREM 5. Let $A_{i}(1 \leq i \leq m)$ be positive definite operators in $C_{1}$; then

$$
\left|\operatorname{Tr}\left(A_{1} A_{2} \cdots A_{m}\right)\right|<\prod_{i=1}^{m} \operatorname{Tr}\left(A_{i}\right), \quad m \geq 2 .
$$

Proof. By Lemma 2 and Lemma 4, we get

$$
\begin{aligned}
& \left|\operatorname{Tr}\left(A_{1} A_{2} \cdots A_{m}\right)\right|=\left|\operatorname{Tr}\left[A_{1}\left(A_{2} \cdots A_{m}\right)\right]\right| \\
& \quad \leq \sqrt{\operatorname{Tr}\left(A_{1} A_{1}^{*}\right)} \cdot \sqrt{\operatorname{Tr}\left[\left(A_{2} \cdots A_{m}\right)\left(A_{2} \cdots A_{m}\right)^{*}\right]}<\prod_{i=1}^{m} \operatorname{Tr}\left(A_{i}\right)
\end{aligned}
$$

The proof is complete.

THEOREM 6. Let $A_{i}(1 \leq i \leq m)$ be positive definite operators in $C_{1}$; then

$$
\frac{1}{m}\left[\sum_{i=1}^{m} \operatorname{Tr}\left(A_{i}\right)\right]>\left|\operatorname{Tr}\left(A_{1} A_{2} \cdots A_{m}\right)\right|^{\frac{1}{m}} .
$$

PROOF. By the arithmetic mean - geometric mean inequality for $m$ positive real numbers, we have

$$
\frac{1}{m}\left[\sum_{i=1}^{m} \operatorname{Tr}\left(A_{i}\right)\right] \geq\left(\prod_{i=1}^{m} \operatorname{Tr}\left(A_{i}\right)\right)^{\frac{1}{m}}
$$

From Theorem 5, we get

$$
\frac{1}{m}\left[\sum_{i=1}^{m} \operatorname{Tr}\left(A_{i}\right)\right]>\left|\operatorname{Tr}\left(A_{1} A_{2} \cdots A_{m}\right)\right|^{\frac{1}{m}} .
$$

The proof is complete. 
The above Theorem 6 generalizes the theorem in [3].

THEOREM 7. Let $A_{i}\left(1 \leq i \leq 2^{m}\right)$ be positive definite operators in $C_{1}$; then

$$
\left|\operatorname{Tr}\left(A_{1} A_{2} \cdots A_{2^{m}}\right)\right|^{2^{m}} \leq \prod_{i=1}^{2^{m}}\left[\operatorname{Tr}\left(A_{i}^{2^{m}}\right)\right]
$$

ProOF. We will prove the above inequality by induction on $m$. If $m=1$, inequality (2) is obvious by Lemma 2 . Now suppose that, for $m<p$, inequality (2) is true. If $m=p$, let

$$
\begin{aligned}
B_{i} & =A_{2^{p-i}} A_{2^{p-i}-1} \cdots A_{2} A_{1} A_{1} A_{2} \cdots A_{2^{p-i}-1} A_{2^{p-i}} \\
\text { and } \quad C_{i} & =A_{2^{p-i}+1} A_{2^{p-i}} \cdots A_{2^{p-(i-1)}} A_{2^{p-(i-1)} \cdots A_{2^{p-i}} A_{2^{p-i}+1}} \\
u_{i} & =\left|\operatorname{Tr}\left(B_{i}^{2^{i-1}}\right)\right|^{2^{p-i}}, \quad v_{i}=\left|\operatorname{Tr}\left(C_{i}^{2^{i-1}}\right)\right|^{2^{p-i}}, \quad 1 \leq i \leq p .
\end{aligned}
$$

We have

$$
\begin{aligned}
u_{i}= & \left|\operatorname{Tr}\left(B_{i}^{2^{i-1}}\right)\right|^{2^{p-i}} \\
= & \operatorname{Tr}\left[\left(A_{2^{p-i}} \cdots A_{1}^{2} \cdots A_{2^{p-i}}\right)\left(A_{2^{p-i}} \cdots A_{1}^{2} \cdots A_{2^{p-i}}\right) \cdots\right. \\
& \left.\quad\left(A_{2^{p-i}} \cdots A_{1}^{2} \cdots A_{2^{p-i}}\right)\right]\left.\right|^{2^{p-i}} \quad\left(\text { with } 2^{i-1}\right. \text { bracketed factors) } \\
= & \mid \operatorname{Tr}\left[\left(A_{2^{p-i-1}} \cdots A_{1}^{2} \cdots A_{2^{p-i-1}}\right)\left(A_{2^{p-i-1}+1} \cdots A_{2^{p-i}}^{2} \cdots A_{2^{p-i-1}+1}\right) \cdots\right. \\
& \left.\quad\left(A_{2^{p-i-1}} \cdots A_{1}^{2} \cdots A_{2^{p-i-1}}\right)\left(A_{2^{p-i-1}+1} \cdots A_{2^{p-i}}^{2} \cdots A_{2^{p-i-1}+1}\right)\right]\left.\right|^{2^{p-i}} \\
& \quad\left|\operatorname{Tr}\left(B_{i+1} C_{i+1} \cdots B_{i+1} C_{i+1}\right)\right|^{2^{p-i}}, \quad(i \leq p-1) \quad\left(2^{i-1} \text { factors } B_{i+1} C_{i+1}\right) \\
\leq & \left|\left[\prod_{j=1}^{2^{i-1}}\left\{\operatorname{Tr}\left(B_{i+1}^{2^{i}}\right) \operatorname{Tr}\left(C_{i+1}^{2^{i}}\right)\right\}^{2^{i}}\right]\right|^{2^{p-i}} \quad \text { (by inductive hypothesis) } \\
= & \left|\operatorname{Tr}\left(B_{i+1}^{2^{i}}\right)\right|^{2^{p-i-1}} \cdot\left|\operatorname{Tr}\left(C_{i+1}^{2^{i}}\right)\right|^{2^{p-i-1}} \\
\leq & u_{i+1} \cdot v_{i+1} \quad(1 \leq i<p)
\end{aligned}
$$

that is, $u_{i} \leq u_{i+1} \cdot v_{i+1}, 1 \leq i<p$.

Since

$$
u_{p-1}=\left|\operatorname{Tr}\left(B_{p-1}^{2^{p-2}}\right)\right|^{2}=\left|\operatorname{Tr}\left\{\left(A_{2} A_{1}^{2} A_{2}\right)^{2^{p-2}}\right\}\right|^{2}
$$


[5]

$$
\begin{aligned}
& =\left|\operatorname{Tr}\left(A_{1}^{2} A_{2}^{2} A_{1}^{2} A_{2}^{2} \cdots A_{1}^{2} A_{2}^{2}\right)\right|^{2} \quad \text { (by Lemma 1) } \quad\left(2^{p-2} \text { factors } A_{1}^{2} A_{2}^{2}\right) \\
& \leq\left|\prod_{i=1}^{2^{p-2}}\left[\operatorname{Tr}\left\{\left(A_{1}^{2}\right)^{2^{p-1}}\right\} \cdot \operatorname{Tr}\left\{\left(A_{2}^{2}\right)^{2^{p-1}}\right]\right]^{\frac{1}{2^{p-1}}}\right|^{2} \quad \text { (by inductive hypothesis) } \\
& =\operatorname{Tr}\left(A_{1}^{2^{p}}\right) \operatorname{Tr}\left(A_{2}^{2^{p}}\right),
\end{aligned}
$$

and

$$
\begin{aligned}
v_{p-1} & =\left|\operatorname{Tr}\left(C_{p-1}^{2^{p-2}}\right)\right|^{2}=\left|\operatorname{Tr}\left(A_{3} A_{4}^{2} A_{3}^{2} A_{4}^{2} \cdots A_{4}^{2} A_{3}\right)\right|^{2} \\
& =\left|\operatorname{Tr}\left(A_{3}^{2} A_{4}^{2} \cdots A_{3}^{2} A_{4}^{2}\right)\right|^{2} \quad \text { (by Lemma 1) } \\
& \leq\left|\prod_{i=1}^{2^{p-2}}\left[\operatorname{Tr}\left\{\left(A_{3}^{2}\right)^{2^{p-1}}\right\} \cdot \operatorname{Tr}\left\{\left(A_{4}^{2}\right)^{2^{p-1}}\right\}\right]^{\frac{1}{2^{p-1}}}\right|^{2} \\
& =\operatorname{Tr}\left(A_{3}^{2^{p}}\right) \operatorname{Tr}\left(A_{4}^{2^{p}}\right),
\end{aligned}
$$

(by inductive hypothesis)

we have

$$
u_{p-2} \leq u_{p-1} \cdot v_{p-1} \leq \operatorname{Tr}\left(A_{1}^{2^{p}}\right) \operatorname{Tr}\left(A_{2}^{2^{p}}\right) \operatorname{Tr}\left(A_{3}^{2^{p}}\right) \operatorname{Tr}\left(A_{4}^{2^{p}}\right) .
$$

In exactly the same way, we can establish the following inequality.

$$
\begin{aligned}
& v_{p-2} \leq \prod_{i=5}^{8} \operatorname{Tr}\left(A_{i}^{2^{p}}\right) \\
& u_{p-3} \leq u_{p-2} v_{p-2} \leq \prod_{i=1}^{8} \operatorname{Tr}\left(A_{i}^{2^{p}}\right) \\
& \ldots \\
& u_{1} \leq \prod_{i=1}^{2^{p-1}} \operatorname{Tr}\left(A_{i}^{2^{p}}\right) \\
& v_{1} \leq \prod_{i=2^{p-1}+1}^{2^{p}} \operatorname{Tr}\left(A_{i}^{2^{p}}\right) .
\end{aligned}
$$

Therefore we obtain

$$
\begin{aligned}
& \left|\operatorname{Tr}\left(A_{1} A_{2} \cdots A_{2^{p}}\right)\right|^{2^{p}}=\left|\operatorname{Tr}\left[\left(A_{1} A_{2} \cdots A_{2^{p-1}}\right)\left(A_{2^{p-1}+1} \cdots A_{2^{p}}\right)\right]\right|^{p^{p}} \\
& \quad \leq\left|\operatorname{Tr}\left(A_{2^{p-1}} \cdots A_{1}^{2} \cdots A_{2^{p-1}}\right) \cdot \operatorname{Tr}\left(A_{2^{p-1}+1} \cdots A_{2^{p}}^{2} \cdots A_{2^{p-1}+1}\right)\right|^{2^{p-1}} \\
& \quad=u_{1} \cdot v_{1} \leq \prod_{i=1}^{2^{p}} \operatorname{Tr}\left(A_{i}^{2^{p}}\right) .
\end{aligned}
$$


Finally, we present an open question:

Let $A_{i}(1 \leq i \leq m)$ be positive definite operators in $C_{1}$. Does the following inequality hold:

$$
\left|\operatorname{Tr}\left(A_{1} A_{2} \cdots A_{m}\right)\right|^{m} \leq \prod_{i=1}^{m}\left[\operatorname{Tr}\left(A_{i}^{m}\right)\right] \quad ?
$$

\section{References}

[1] R. Bellman, 'Some inequalities for positive matrices', in: General inequalities 2. Proceedings, 2nd International Conference on General Inequalities (ed. E. F. Backenbach), (Birkhauser, Basel, 1980) pp. 89-90.

[2] J. R. Ringrose, Compact non-self-adjoint operators (Van Nostrand, New York, 1971).

[3] D. Singh, 'A trace inequality for operators', J.Math.Anal.Appl. 150 (1990), 159-160.

[4] Y. Yang, 'A matrix trace inequality', J.Math. Anal. Appl. 133 (1988), 573-574.

\section{Department of Mathematics}

Chongqing Normal University

Chongqing, 630047

CHINA 